\title{
Article
}

\section{Santé reproductive des adolescents en Afrique : pour une approche globale}

\author{
Josiane Carine Tantchou Yakam \\ Anthropologue, INSERM-IRD-U2, Observatoire régional de la santé (PACA), UMR 912, Sciences économiques et sociales, \\ systèmes de santé, sociétés, 13006 Marseille, France
}

\begin{abstract}
La santé reproductive est un sujet d'intérêt pour la revue NSS surtout lorsqu'il s'agit d'aborder cette question par une approche globale. La reproduction humaine est un processus biologique qui est en effet devenu une question très médicalisée en même temps qu'elle reste une dimension s'inscrivant dans le social et le culturel. C'est donc bien au croisement de la biologie et de la culture que s'opère cette approche de la santé reproductive des adolescents, ce qui suppose un dialogue entre sciences biomédicales et sciences sociales. Cette dimension prend encore plus d'importance avec l'épidémie de sida comme l'avait déjà souligné Marc Eric Gruénais dans un dossier de notre revue sur la Santé $(N S S, 9,4)$. Josiane Tantchou revient sur cette question et discute comment les adolescents africains perçoivent les risques liés à la sexualité (contraception, grossesse, avortement...) dans un contexte spécifique de pauvreté et de violence.
\end{abstract}

La Rédaction

\section{Mots-clés :}

adolescent :

sexualité ;

risque;

facteurs structurels; Afrique

\section{Keywords:}

adolescents;

sexuality;

risk;

structural factors;

Africa
Résumé - Le présent article est une synthèse de la littérature socio-anthropologique et démographique sur la santé reproductive des adolescents en Afrique. Son objectif est de proposer une approche globale du comportement sexuel des adolescents, en mettant en évidence les facteurs structurels susceptibles de l'influencer. La littérature a été réunie à partir des bases de données JSTOR et Medline, consultées en 2006 et 2008. Cette recension met en évidence la vulnérabilité des adolescentes, évoluant dans des contextes de précarité, d'inégalité de genre et de violence sexuelle; elle suggère que le comportement sexuel des adolescents est tributaire de facteurs contextuels que les analyses devraient prendre en compte. Le texte s'articule en trois parties. Il traite d'abord des principaux problèmes associés à l'activité sexuelle des adolescents ; sont ensuite évoquées les stratégies employées pour faire face à ces problèmes et leurs limites. Enfin, dans la dernière partie, l'auteur revient sur quelques facteurs structurels qui influencent le comportement sexuel des adolescents.

\begin{abstract}
For a comprehensive approach to reproductive health of adolescents in Africa. The paper reviews literature on adolescent reproductive health in Africa. It aims to highlight the structural factors which shape their sexual behaviour. The literature was collected using JSTOR and Medline databases. We first entered the words "reproductive health" to get an overall idea of existing literature. The field was then narrowed by entering: "reproductive health, youth", "reproductive health, youth, Africa", "youth, sexuality, Africa". We looked into the references provided in the articles. The paper is structured in three parts. The first presents the major problems associated with young people's sexual behaviour. The second deals with solutions used to tackle these problems. The third concerns the structural factors which shape young people's sexual behaviour. It focuses especially on economic factors, gender inequalities and sexual violence. We conclude that: studies undertaken on reproductive health of adolescents especially highlight the vulnerability of girls ; adolescents' sexual behaviour cannot be separated from their social, cultural, political and economic environment.
\end{abstract}

Auteur correspondant : Josiane.Tantchou@ird.fr 


\section{Introduction}

Depuis la Conférence internationale du Caire sur la population et le développement (1994), l'accès des adolescents aux services de santé reproductive ${ }^{1}$ est entré dans les agendas internationaux.

L'adolescence est une période de transition, de maturation physique, de formation de l'identité et d'acquisition des rôles sociaux qui est associée à l'émergence d'une conscience de la sexualité et du désir de l'expérimenter (Dehne et Riedner, 2001). À cette période de leur vie, les jeunes sont particulièrement sensibles à la pression des pairs et peuvent, en jugeant de façon irrationnelle les avantages et les inconvénients de certains comportements, prendre des décisions mettant leur vie en danger. Or, la sexualité est perçue avec beaucoup d'ambiguïté dans la plupart des régions du monde. En effet, si on admet que les adolescents puissent avoir une vie sexuelle, elle est souvent jugée problématique à cause des risques encourus : infections sexuellement transmissibles (IST), grossesses non désirées, et les prohibitions sociales imposées aux adultes sont accrues chez les adolescents. Toutefois, en insistant sur les risques que l'épidémie de $\mathrm{VIH} /$ sida est venue renforcer, on occulte l'expérience même de la sexualité : expérimentation de l'émergence du désir, stratégies pour le gérer, le contrôler, négociation des rapports sexuels (ibid.).

La découverte des liens de causalité entre les agents de maladies et les comportements a déplacé la responsabilité de la prévention de la société vers l'individu. La maladie est passée d'un danger toujours présent à un risque lié au style de vie. Ainsi évoque-t-on les comportements à risque, les situations à risque, les populations à risque, ces dernières étant la plupart du temps «assumed rather than revealed» (Standing, 1992). Dans le cadre de la prévention du $\mathrm{VIH} /$ sida, ont été visés les prostitués, les routiers, les «hommes en tenue » (corps de l'armée), les adolescents. Cette approche, individualisante, soutient l'idée d'une liberté de choix et d'une citoyenneté sexuelle dont certaines catégories de population sont pourtant privées. La focalisation sur l'individu occulte les facteurs structurels (écologiques, environnementaux, contextuels) qui influencent le comportement sexuel. Ce texte propose une analyse critique de l'approche individualisante et plaide pour une approche globale. Nous souhaitons relever, à partir de la littérature ${ }^{2}$, les facteurs structurels

\footnotetext{
${ }^{1}$ La Conférence du Caire (organisée sous les auspices de l'ONU, du 5 au 13 septembre 1994) en donne la définition suivante: "Reproductive health is a state of complete physical, mental and social well-being and not merely the absence of disease or infirmity, in all matters relating to the reproductive system and to its functions and processes. Reproductive health therefore implies that people are able to have a satisfying and safe sex life and that they have the capability to reproduce and the freedom to decide if, when and how often to do so » (Glasier et al., 2006).

${ }^{2}$ Réalisée dans le cadre du programme d'Amélioration de la qualité et de l'accès aux soins obstétricaux d'urgence dans les
}

qui influencent le comportement sexuel des adolescents, participent à la construction des situations de risque et limitent l'adoption de comportements « sains ».

La bibliographie a été constituée à partir des bases de données JSTOR et Medline. Nous nous sommes surtout intéressée aux textes publiés à partir des années 1990 et, exceptionnellement, à la fin des années 1980. Sur JSTOR, nous avons, dans un premier temps, rentré les mots "reproductive health» afin d'avoir une idée de l'ensemble des travaux sur la question. Nous avons ensuite restreint le champ en rentrant les termes : "adolescents sexuality», "adolescents sexuality, Africa», "reproductive health, youth", "reproductive health, youth, Africa », «youth, sexuality, Africa». Les bases de données ont été consultées de mars à juin et de septembre à octobre 2006, puis de janvier à février 2008. Nous avons aussi retenu certaines recherches menées en Europe, en Amérique ou en Australie qui éclairent le contexte africain. Il s'agit des travaux de Geronimus (1991, 1992 et 2004), Tolman (1994), Hanna, (2001), Gavey et al., (2001), Scott-Sheldon et al. (2006), Shoveller et al., (2004). Nous avons noté que peu d'études se consacrent exclusivement aux adolescents (les 10-19 ans) et que cette catégorie reste très ambiguë. Selon l'Organisation mondiale de la santé (OMS) ${ }^{3}$, est adolescent tout individu dont l'âge est compris entre 10 et 19 ans. La catégorie «adolescent » chevauche celle des jeunes (15-24 ans) et la "population jeune » englobe les individus de 10 à 24 ans. Dans la littérature, dominée par les organisations internationales, l'adolescence est située selon les cas entre 15-19 ans, 15-24 ans, 10-19 ans ou 10-24 ans (Dehne et Riedner, 2001). Dans les travaux de recherche, les auteurs retiennent des fourchettes différentes : «young people» pour les 14-19 ans ou les 12-26 ans, «youth» pour les 14-21 ans, "young men and women " pour les 17-25 ans , «men» s'agissant des 15-26 ans, «jeunes» de 10 à 24 ans (Guiella, 2004), voire « adolescents » sans précision d'âge. Les catégories utilisées dans la plupart des travaux excluent les 10-13 ans et les raisons qui ont conduit à inclure ou exclure certaines tranches d'âge sont rarement indiquées. Ce constat montre que les travaux de recherche relatifs aux adolescents vont au-delà de la catégorie telle qu'elle est définie par l'OMS. Pour notre étude, nous nous sommes donc intéressée à la littérature sur la «population jeune » au sens le plus large (Guiella, 2004), en accordant une attention particulière aux travaux portant sur ou incluant les 10-19 ans.

Le présent texte est structuré en trois parties. Il traite d'abord des principaux problèmes associés à l'activité sexuelle des adolescents, puis des mesures mises en œuvre pour faire face à ceux-ci, ainsi que de leurs limites. La

pays en développement (AQUASOU). Projet FSP 2001-149. Nous tenons à remercier les lecteurs de NSS pour leurs commentaires qui ont permis de mieux préciser l'objectif de ce texte.

${ }^{3}$ Cf.http://www.who.int/child-adolescent-health/OVERVIEW/ AHD/adh_over.htm. 
troisième partie relève quelques facteurs structurels qui influencent le comportement sexuel.

\section{Grossesses, avortements, IST/VIH : le trio menaçant}

En dépit du sentiment de gêne que les adultes peuvent éprouver à cette idée, leurs enfants adolescents sont sexuellement actifs ou ont eu des rapports sexuels avant l'âge adulte (Shoveller et al., 2004). La curiosité, l'expérience, les raisons économiques, les pulsions naturelles, l'amour, une promesse de mariage, la pression des pairs, la peur de la violence, le viol sont évoqués pour commencer une activité sexuelle (Meekers et Calvès, 1997; Wood et al., 1998; Rwenge, 2000 ; Gueye et al., 2001; Nyanzi et al., 2001 ; Afenyadu et Goparaju, 2003; Guiella, 2004 ; Koenig et al., 2004; Amuyunzu-Nyamongo et al., 2005). Afin d'éviter les IST, y compris le VIH/sida, et/ou les grossesses non désirées, il est généralement demandé aux jeunes sexuellement actifs d'adopter des " comportements sains » ou «sans risque » tels que l'abstinence, la fidélité à son ou sa partenaire, l'utilisation des préservatifs lors des rapports sexuels occasionnels ${ }^{4}, l^{\prime}$ utilisation d'autres méthodes contraceptives dont la pilule. Cependant, plusieurs facteurs conduisent les jeunes à ne pas suivre ces recommandations, quand bien même ils auraient été «sensibilisés ».

\section{Les grossesses d'adolescentes}

Dans le monde, environ 14 millions d'adolescentes deviennent mères chaque année et près de $90 \%$ de celles-ci vivent dans les pays en voie de développement (Reynolds et al., 2006). Les pays d'Afrique subsaharienne ont les taux de maternité d'adolescentes les plus élevés au monde. Diverses explications sont avancées par les chercheurs : mariages précoces, besoin de prouver sa fertilité, ignorance, acceptation réduite des méthodes contraceptives «modernes», rareté des services de planning familial, séduction des «sugar-daddies ${ }^{5} »$. Les grossesses chez des filles qui n'ont pas achevé leur développement physiologique et physique ont des répercussions sur la santé de la mère et de $1^{\prime}$ enfant ${ }^{6}$, mais aussi des conséquences

\footnotetext{
${ }^{4}$ Dans le cadre de la prévention de la transmission du VIH, il est recommandé d'arrêter l'usage des préservatifs lorsque les deux partenaires ont fait le dépistage deux fois à trois mois d'intervalle (pour éliminer le risque d'être en primo-infection lors du premier test) et que les résultats des dépistages sont bien négatifs.

${ }^{5}$ Hommes plus âgés et d'un statut social plus élevé : voir, plus loin, le paragraphe sur le facteur économique.

${ }^{6}$ L'immaturité du squelette au niveau de la zone pelvienne augmente les risques d'accouchements difficiles : travail prolongé, etc. Les adolescentes n'ont aucune expérience de la
}

sociales : si elle est scolarisée, l'adolescente est parfois obligée d'interrompre son cursus scolaire, ce qui réduit ses perspectives d'avenir, notamment celle de trouver un emploi stable. Ces grossesses non planifiées sont à l'origine de déception, de conflits familiaux et/ou matrimoniaux. La jeune mère s'expose à l'opprobre, court le risque de rester célibataire ou de devoir épouser l'auteur de la grossesse (Gorgen et al., 1993; Schwab Zabin et Kiragu, 1998 ; Calvès, 1999 ; Afenyadu et Goparaju, 2003 ; Amuyunzu-Nyamongo et al., 2005) ${ }^{7}$. Précisons toutefois que les conséquences peuvent être très différentes selon les situations (par exemple, une grossesse chez une femme mariée de 19 ans ou une grossesse «illégitime » chez une jeune fille de 17 ans).

Les grossesses d'adolescentes sont généralement perçues comme un «problème social », car elles sont associées à la pauvreté, à la déscolarisation qui accompagne potentiellement la grossesse et aux difficultés de carrière qui en résultent. L'adolescente enceinte est alors présentée comme une menace pour la sécurité économique. Arline Geronimus souligne qu'aux États-Unis, les adolescentes mères viennent préférentiellement de familles défavorisées, appartiennent à des groupes minoritaires, ont été élevées dans des banlieues ou des communautés rurales extrêmement isolées. Elles appartiennent donc à des groupes ou habitent des régions qui, sur les plans scolaire et sanitaire, sont mal ou pas desservies et ont par conséquent des opportunités plus réduites d'accès à une certaine aisance matérielle. Est-il, dans ces conditions, pertinent d'établir une relation entre le statut socioéconomique ou la mauvaise santé et l'âge de la première grossesse? La maternité est-elle réellement à l'origine des problèmes socioéconomiques auxquels sont confrontées les adolescentes mères, s'interroge A. Geronimus? Elle constate qu'on n'évoque guère les adolescentes qui donnent naissance à des enfants en bonne santé et retournent à l'école, parviennent à un statut social et économique respectable, se marient et ont d'autres enfants. Tout l'intérêt semble porter sur celles pour lesquelles la grossesse est supposée marquer le commencement d'un enchaînement de difficultés. Par ailleurs, les politiques et les programmes destinés à prévenir les grossesses chez les adolescentes occultent complètement le rôle des hommes , renforçant la représentation selon laquelle les jeunes filles

maternité, elles ne sont pas armées psychologiquement et émotionnellement pour faire face et cela a des répercussions sur l'alimentation et la santé des nourrissons. Nous soulignerons par ailleurs que les enfants nés d'adolescentes ont des poids inférieurs à ceux des enfants nés de mères plus âgées, ce qui laisse supposer une plus grande fragilité (LeGrand et Mbacké, 1993).

Amuyunzu-Nyamongo et al. (2005) ont constaté que les adolescents ne sont pas jugés aussi sévèrement. Raillés de temps en temps, ils sont aussi perçus comme de «vrais hommes » et admirés. Ils peuvent nier leur responsabilité, chose impossible aux mères. 
sont les principales coupables. Comme le remarque Varga (ibid.), "both research and programming have traditionally been dominated by the assumption that sexual and reproductive health issues are primarily female concerns ». En outre, les grossesses précoces d'adolescentes sont souvent considérées comme involontaires. Or, Hanna (2001) montre qu'en Australie, elles peuvent parfois être planifiées dans la mesure où ces adolescentes désirent accéder, par la maternité, au statut d'adulte et à un certain accomplissement. Des recherches menées en Afrique ont également montré que certaines adolescentes ne refusaient pas l'éventualité d'une grossesse, mais s'interrogeaient sur le moment propice.

Au regard des éléments avancés, on peut se demander, comme A. Geronimus (2004), si la vision selon laquelle les grossesses d'adolescentes sont un «problème social » ne peut être interprétée comme un engagement de la «culture dominante » à promouvoir ses propres valeurs. En effet, l'idéal familial blanc américain exige que les parents biologiques soient autosuffisants (Geronimus, 1991). Aînés et parents emploient alors les espaces publics (médias, associations militantes, campagnes politiques, programmes scolaires, financement de la recherche scientifique et publications) pour alerter leurs enfants sur les conséquences désastreuses des grossesses précoces. Si la situation est différente en Afrique, l'argument mérite tout de même d'être examiné. D'une part, il existe une «culture de censure politique et de peur » dont Horton (2006) remarque qu'elle se répand dans plusieurs institutions de santé publique quand il est question de santé reproductive ; d'autre part, les organisations internationales qui définissent l'orientation de la plupart des politiques sanitaires menées en Afrique ont une influence déterminante. L'USAID (United States Agency for International Development), par exemple, a imposé pendant une certaine période des messages de prévention insistant plutôt sur l'abstinence et la fidélité que sur l'usage du préservatif comme condition de financement des programmes nationaux de lutte contre le sida. Alors que pendant de nombreuses années elle a été le principal exportateur de préservatifs dans les pays africains, l'USAID était opposée à leur distribution aux adolescents en dehors du mariage, «obligeant » même ses partenaires à adopter la même politique en menaçant de supprimer son aide. Devant les conséquences réelles ou présumées d'une naissance à venir, et en l'absence de contraception moderne ou efficace, les adolescentes mettent souvent un terme aux grossesses en ayant recours à des pratiques abortives.

\section{L'avortement : pratique illégale, clandestine, dangereuse, mais courante}

Il n'y a pas de données officielles sur l'avortement en Afrique subsaharienne. Les taux semblent cependant très élevés et en continuelle progression, surtout chez les jeunes filles célibataires. Les raisons évoquées pour l'avortement sont le désir de finir ses études, le caractère instable de la relation, le déni de la paternité, le sentiment d'être trop jeune pour avoir un enfant, la pauvreté, la peur de la réaction des parents.

Interdits par la loi, les avortements sont réalisés «clandestinement » par des médecins ou des infirmières, des étudiants en médecine, des sages-femmes ou par une variété de praticiens sans formation précise (Barker et Rich, 1992 ; Gorgen et al., 1993 ; Calvès, 2002). Ils sont pratiqués à domicile ou dans les structures sanitaires privées ou publiques. Les filles ne recourent pas systématiquement aux « spécialistes » et emploient diverses substances pour expulser le fœetus (Lallement et Jourdain, 1985 ; Gorgen et al. 1993) : permanganate de potassium, doses importantes de chloroquine ou de paracétamol ${ }^{8}$, grande quantité de café en poudre, miel sans autre repas pendant plusieurs jours, fortes doses de sucre et de citron, éclats de verre écrasés et insérés dans le vagin. Conscientes des risques auxquels elles s'exposent, quelques-unes perçoivent l'avortement comme un fait de la vie comportant des risques inévitables (Barker et Rich, 1992).

En plus du risque de grossesse qui peut donner lieu à un avortement, mettant en danger la vie et la fertilité de la jeune mère, les adolescent(e)s sont aussi expos(é)es au risque de contracter une IST ou le VIH.

\section{IST/VIH}

Il est difficile d'appréhender avec exactitude l'ampleur des IST, considérées comme des maladies honteuses (Guiella, 2004). La plupart des adolescents sexuellement actifs indiquent qu'ils n'ont jamais eu d'IST , mais leurs connaissances en ce domaine sont limitées. Afenyadu et Goparaju (2003) ont relevé deux IST principalement connues des adolescents du Ghana : la blennorragie et le $\mathrm{VIH} /$ sida. En Afrique du Sud, les jeunes citent différents types d'IST, mais ignorent les modes réels de transmission (Barker et Rich, 1992); au Burkina Faso et au Ghana, la bilharziose ${ }^{9}$ a été classée dans la catégorie des IST (Amuyunzu-Nyamongo et al., 2005).

\footnotetext{
${ }^{8}$ La chloroquine est un antipaludéen. C'est, avec la quinine, le traitement qui a été le plus employé en prévention et comme remède contre le paludisme. Le paracétamol, également appelé acétaminophène, est un médicament de la classe des antalgiques antipyrétiques non salicylés. Il est ainsi utilisé contre la fièvre et la douleur.

9 D'après les auteurs de l'étude, l'identification de cette maladie comme IST peut être due à la façon dont les modérateurs ont introduit la discussion en langue locale. Au Burkina Faso, chez les Mooré, les IST sont dites "illnesses of the front ", front incluant les parties génitales, mais aussi les intestins et le foie ; la bilharziose a peut-être été mentionnée à cause de cette représentation.
} 
En cas d'IST, les jeunes adoptent diverses attitudes. Au Ghana, 59 \% des adolescents qui ont manifesté des symptômes ont affirmé avoir recherché un traitement, mais les auteurs ne précisent pas où (Afenyadu et Goparaju, 2003). Au Burkina Faso, d'après l'enquête démographique et de santé (EDS), $59 \%$ des hommes et $72 \%$ des femmes de 15 à 49 ans ont recherché un traitement auprès du personnel de santé, et seulement $36 \%$ des hommes et $85 \%$ des femmes ont déclaré avoir informé leur partenaire. Une autre étude, menée à Ouagadougou et à Tenkodogo, a révélé que, dans la première de ces villes, seuls $15 \%$ des adolescents interrogés consulteraient les services de santé modernes en cas d'IST, contre $7 \%$ dans la seconde. Par contre, 31 \% des adolescents à Ouagadougou ont déclaré qu'ils consulteraient un tradipraticien ou pratiqueraient l'automédication et $74 \%$ des adolescents de Tenkodogo ont affirmé qu'ils auraient recours à l'automédication. Comme les auteurs de l'étude le précisent, il s'agit d'intentions qui ne peuvent toutefois pas permettre de prédire les comportements (Guiella, 2004).

En 2001, la déclaration des Nations unies sur le $\mathrm{VIH} /$ sida $^{10}$ avait fixé l'objectif de diminuer de $25 \%$ la prévalence du VIH parmi les jeunes (15 à 24 ans) d'ici à 2005, dans les pays les plus touchés, en suivant l'évolution des nouvelles infections. D'après les estimations d'ONUSIDA et de l'OMS, plus de 4 millions de jeunes de moins de 15 ans ont été infectés depuis le début de l'épidémie. Parmi eux, plus de $90 \%$ étaient nés de mères séropositives et ont été infectés pendant l'accouchement ou par le lait maternel. Parce que l'infection progresse rapidement chez les enfants, la majorité des moins de 15 ans contaminés ont développé la maladie et sont décédés. On estime que 10,3 millions de jeunes âgés de 15 à 24 ans vivent avec le $\mathrm{VIH} /$ sida et la moitié de toutes les nouvelles infections (environ 7000 par jour) survient parmi les jeunes ${ }^{11}$. La prévalence chez les femmes est toujours nettement plus élevée : 12 à 13 femmes pour 10 hommes sont infectées par le VIH et la moyenne des taux d'infection chez les filles est, dans certains pays, cinq fois plus élevée que chez les garçons (Gupta, 2002). 57 \% des adultes séropositifs sont des femmes et les jeunes femmes âgées de 15 à 24 ans sont trois fois plus susceptibles d'être infectées que les jeunes hommes (Kim et Watts, 2005). Le récent Point sur l'épidémie de sida relève cependant que, pour 11 des 15 pays les plus touchés par la maladie et disposant de données suffisantes (trois années différentes), la prévalence du VIH chez les jeunes femmes enceintes de 15 à 24 ans, vues en consultation prénatale, a diminué depuis 2000-2001. Par contre, en Afrique du Sud, au Mozambique et en Zambie, aucun signe de décroissance de l'infection parmi les jeunes n'est observé.

\footnotetext{
${ }_{10}$ Cf. http://data.unaids.org/publications/irc-pub03/aidsdeclaration_en.pdf

${ }^{11}$ Cf. http://www.who.int/child-adolescent-health/over.htm (consulté le 7 janvier 2007).
}

Les recherches menées par Amuyunzu-Nyamongo et al. (2005) montrent que les jeunes ont des connaissances plus approfondies sur le VIH/sida que sur les autres IST. Néanmoins, des préjugés persistent. Au Burkina Faso, $35 \%$ des filles et $26 \%$ des garçons de 15 à 19 ans ne connaissent aucun moyen d'éviter le sida. $20 \%$ des jeunes filles et $3 \%$ des jeunes garçons de 15 à 19 ans pensent qu' « éviter d'embrasser », " éviter de boire et manger dans la même vaisselle qu'un sidéen » permettent de s'en prémunir, tandis que $9 \%$ des $15-24$ ans pensent que le sida ne peut pas être évité. En milieu rural, certains adolescents continuent de croire que les moustiques peuvent transmettre le virus du sida (Guiella, 2004). AmuyunzuNyamongo et al. (2005) ont fait des constats semblables et ajoutent que certains adolescents n'établissent pas de différences entre le VIH et le sida.

\section{L'éducation, le préservatif : des stratégies limitées}

\section{L'éducation sexuelle}

Le constat général est celui d'une absence de dialogue entre parents et adolescents. Les adolescents craignent la réaction des parents s'ils abordent le sujet, éprouvent une certaine gêne à parler de sexualité avec les adultes (Barker et Rich, 1992) et, parfois, ce type de discussion est simplement tabou (Nyanzi et al., 2001 ; AmuyunzuNyamongo et al., 2005). Le sujet est quelquefois traité dans les cours de biologie, mais les adolescents n'y trouvent pas les informations pouvant véritablement leur permettre de comprendre leurs émotions, les mécanismes de la sexualité, prévenir les grossesses, éviter les IST et le sida (Barker et Rich, 1992). Pour la majorité, les pairs, les films et les magazines, dont les documents pornographiques, sont dans cet ordre les principales sources d'information (Amuyunzu-Nyamongo et al., 2005). Dans une étude menée au Ghana, les jeunes ont indiqué qu'ils obtenaient l'information de quatre sources principales : les médias (la radio était évoquée comme source majeure et privilégiée) ; les écoles ou les maîtres ; les personnels de santé ; la famille et les amis. Les ONG, les centres de jeunes, les clubs de jeunes, les leaders religieux ont aussi été cités (ibid.).

Devant le constat d'une absence de dialogue entre parents et adolescents, des programmes d'éducation par les pairs, fondés sur l'idée que les adolescents parlent plus facilement de sexualité avec ceux-ci et que la connaissance entraîne un changement de comportement, ont été développés. Erulkar et al. (2006) ont mené une étude sur la couverture et l'utilisation de ce type de programmes à Addis-Abeba, en Éthiopie. En dépit de leur multiplicité, ces programmes n'atteignent pas véritablement leur cible. Ils s'avèrent surtout adaptés à ceux qui habitent à proximité des centres de jeunes, ont du temps 
libre, des facilités de déplacement. Ceux qui résident dans les quartiers éloignés et ont des obligations domestiques importantes ne sont pas touchés par ces initiatives. Par ailleurs, l'éducation n'entraîne pas nécessairement l'adoption de comportements "sains ». On pourrait, au regard des messages diffusés lors des campagnes d'éducation, affirmer que cela n'est pas surprenant : " restez abstinent », "soyez monogame », " réduisez le nombre de vos partenaires » et «connaissez votre partenaire ». Ces messages ambigus peuvent être interprétés différemment. Ainsi, la monogamie pourrait désigner le fait d'avoir un(e) même partenaire pendant une durée d'une semaine, un mois ou une année - ce que Marshall et Suggs (1970) désignent sous l'expression «serial monogamy».

La littérature sur l'éducation sexuelle des adolescents en Afrique est, dans un certain sens, restée figée sur le constat de l'absence de dialogue entre parents et adolescents. Pourtant, si cela reste vrai dans une certaine mesure, si une éducation sexuelle en tant que programme destiné à informer les jeunes sur toutes les questions relatives à la santé de la reproduction n'existe toujours pas dans plusieurs pays africains, le VIH/sida est venu bouleverser le contexte de prohibition et de silence qui jusque-là était la norme. Entendre les religieux parler de sexualité, écouter les questions de sexualité développées sur les antennes d'une radio nationale sont autant de choses qui, quelques années avant et même après l'apparition du $\mathrm{VIH} /$ sida, étaient inacceptables. L'évolution de la maladie a poussé les responsables d'églises, d'associations, des États à plus de réalisme. Pour prévenir il faut parler, il faut parler de sexe, de sexualité, de rapport sexuel de façon claire et précise. Les projections cinématographiques ou les conférences suivies de débats sur divers thèmes liés à la sexualité sont autant d'occasions qui permettent aux adolescents de s'exprimer et d'approfondir leurs connaissances. Les « semaines culturelles » organisées dans les établissements secondaires constituent aussi des canaux importants de communication. Enfin, les ONG diffusent l'information à travers des dépliants, des affiches. Des campagnes dites «de sensibilisation»sont aussi organisées : "caravanes contre le sida » au Burkina Faso (Guiella, 2004), «vacances sans sida » ou campagne « pincez, déroulez » sur l'utilisation du préservatif au Cameroun. Si celles-ci ne permettent pas vraiment aux adolescents de comprendre les mécanismes de la sexualité, on peut tout de même constater que le temps du silence et de la prohibition est révolu. Notons par ailleurs que les adolescents ont désormais accès, grâce à Internet, à toutes sortes de documents sur les SOW et les $\mathrm{SEW}^{12}$. Les programmes d'éducation doivent s'ajuster à ces changements.

\footnotetext{
12 SOW : sexually oriented websites; SEW : sexually explicit websites.
}

\section{Le préservatif}

Dans les programmes destinés aux adolescents, le préservatif est conseillé pour éviter les grossesses, se protéger des IST et du sida. Or, pour les raisons suivantes, il n'est pas systématiquement utilisé : il diminuerait le plaisir sexuel, suggérerait l'infidélité, la promiscuité, l'absence de confiance; il n'est pas «naturel», il pourrait rester « coincé » dans le vagin. Certains jeunes sont opposés à son utilisation parce qu'ils estiment ne courir aucun risque (Harrison et al., 2001). Si les dernières décennies ont vu une augmentation considérable de son utilisation pour les premières relations sexuelles ou en début de relation, le préservatif est ensuite progressivement abandonné, cette attitude symbolisant le début d'une "relation de confiance ", un nouveau niveau d'engagement. Par ailleurs, lors des relations sexuelles occasionnelles, l'état d'ivresse favorise les rapports sans protection (Singer et al., 2006). L'utilisation des préservatifs dépend aussi de leur disponibilité, de leur accessibilité (dans des conditions qui évitent la gêne ou l'embarras) et de leur coût.

Le Point sur l'épidémie de sida (ONUSIDA et OMS, 2007) indique une évolution contrastée de certains comportements à risque chez les jeunes. La proportion de jeunes qui signalent avoir eu des rapports sexuels avec des partenaires occasionnel(le)s au cours de l'année écoulée a diminué tant chez les hommes que chez les femmes au Kenya, au Malawi et au Zimbabwe. Par contre, elle a augmenté au Cameroun, en Ouganda et au Rwanda. On note également des variations dans le recours au préservatif à cette occasion. Ainsi, la proportion de jeunes déclarant l'avoir utilisé lors du dernier rapport sexuel occasionnel a augmenté chez les deux sexes au Cameroun, au Malawi et en Tanzanie, chez les femmes en Côte d'Ivoire, au Kenya, en Ouganda, au Rwanda et au Togo, mais elle a diminué chez les hommes en Côte d'Ivoire et au Rwanda.

Lors de campagnes de sensibilisation, il est conseillé aux femmes et aux jeunes filles d'exiger le port du préservatif au moins lors de relations sexuelles occasionnelles. Or, proposer un préservatif à son partenaire est un signe de «mœurs légères »; la jeune fille s'expose à la violence et réduit ses chances de construire une relation durable. Plus que l'évitement d'un risque, les femmes privilégient le fait d'être en couple et, même si elles souhaitent que le partenaire mette un préservatif, elles ne «l'ennuieront» pas en le lui suggérant (Singer et al., 2005). En outre, le pouvoir économique, l'autorité symbolique (l'âge du partenaire dans une relation avec un "sugar-daddy ", par exemple) peuvent ruiner toute volonté de négociation, les problèmes financiers supplantant le désir de se protéger d'une maladie hypothétique (Afenyadu et Goparaju, 2003 ; Eaton et al., 2003 ; Amuyunzu-Nyamongo et al., 2005). Ainsi que le soulignait une jeune épouse dans une 
discussion de groupe en Ouganda, le statut matrimonial peut également limiter les capacités de négociation : "You just accept, you have nothing to do because you are married. You have to accept everything he says... now if you are married; your husband might not be faithful to you. You might be faithful but your husband sleeps with an infected person and it becomes a problem... you see the problem you are already married» (Amuyunzu-Nyamongo et al., 2005). Zellner (2003) a montré, pour la Côte d'Ivoire, le rôle déterminant de l'éducation secondaire et supérieure dans l'usage du préservatif.

Néanmoins, d'autres facteurs influencent fortement le comportement sexuel des adolescents et participent à la construction de situations à risque auxquelles il est difficile, voire impossible, $d^{\prime}$ " échapper » ou de «s'échapper ». Notre recherche bibliographique met en évidence les contraintes économiques, les constructions sociales de la masculinité et de la féminité, les inégalités de genre et la violence sexuelle.

\section{Tenir compte du contexte : pauvreté, inégalité de genre, violence sexuelle}

\section{Le facteur économique}

Plusieurs auteurs ont mentionné que les échanges sexuels en Afrique ont une composante financière. Recevoir de l'argent ou des cadeaux d'un homme avec qui on a des rapports sexuels est normal et une fille se sentirait humiliée si elle ne recevait rien en retour ${ }^{13}$, car seule une séropositive peut «donner gratuitement » (Nyanzi et al., 2001). Il faut alors faire la différence entre la jeune fille qui choisit de commencer une activité sexuelle et de recevoir par la suite des cadeaux et celle qui s'y engage pour des raisons économiques. Nyanzi et al. (2001) le mettent bien en évidence pour l'Ouganda. Ici, les adolescentes se sentent embarrassées à l'idée de demander vêtements, chaussures, dessous, accessoires, etc., à leurs parents. Désirer de telles choses suggère une volonté de séduction, et les parents sont incapables de satisfaire ces attentes en raison de leur pauvreté. Plusieurs filles entretiennent de ce fait des rapports sexuels avec des hommes plus âgés et d'un statut social respectable. On parle de «sugar-daddies» (Luke, 2005) ou «uncles » (AmuyunzuNyamongo et al., 2005) pour désigner les hommes qui s'investissent dans ce type de relations («sugar-mummies» pour les femmes); on parle également de «sponsors », de «VVV» (voiture, villa, virement), de «cous pliés ${ }^{14} »$. On

\footnotetext{
${ }^{13}$ Cette constatation fait dire à Standing (1992) que, si le terme prostitution désigne une relation dans laquelle le sexe est échangé contre l'argent, la majorité des unions non maritales en Afrique rentrent dans cette catégorie.

${ }^{14}$ Ils sont souvent obèses, ce qui est perçu comme un signe de bien-être socioéconomique (Meekers et Calvès, 1997).
}

évoque le «sugar-daddy syndrome», le «sugar-daddy trap ». Fragilisée par sa situation économique, une jeune fille ne résistera pas longtemps aux avances d'un «sugar-daddy » qui piège (trap) la jeune adolescente contrainte à la survie ou désireuse d'améliorer ses conditions de vie (ibid.).

On peut, à la lecture de ce qui précède, conclure que les filles ne sont pas seulement des «victimes". Certaines prennent la décision de «sortir» avec un «sugardaddy » car il apporte, en plus de la sécurité économique et d'un support matériel, une expérience et une plus grande satisfaction sexuelles, il peut se marier, prendre en charge une grossesse ou faciliter un avortement, il n'est ni possessif ni aussi jaloux qu'un partenaire plus jeune, il a un appétit sexuel moins important (Meekers et Calvès 1997 ; Nyanzi et al., 2001). Gorgen et al. (1993) ont montré que les filles recherchaient ce type de relation afin de se faire valoir auprès de leurs pairs. En effet, seul un adulte d'un statut économique respectable peut offrir des biens convoités et valorisés par les pairs. Nyanzi et al. (2001) relèvent d'ailleurs que, confrontées au choix entre un collégien et un «sugar-daddy », elles choisiraient ce dernier. Meekers et Calvès (1997) soulignent qu'au Cameroun, les garçons se plaignent $d u$ fait que les filles soient, de façon prédominante, intéressées par les aspects financiers d'une relation, ce qui, selon eux, rend les relations amoureuses coûteuses et instables.

Contrairement à ce qu'on pourrait attendre, les parents ne s'opposent pas nécessairement à ce type de relations et les adolescentes y sont parfois « encouragées ». Des discussions de groupes au Malawi ont révélé que les parents poussaient leurs filles à avoir des rapports sexuels avec des hommes plus âgés et nantis, l'argent obtenu permettant de pourvoir aux besoins de la famille (Amuyunzu-Nyamongo et al., 2005). Les filles se «servent» même de ces «sugar-daddies » à travers la pratique dite «detoothing » (ibid.), qui consiste à obtenir le maximum d'argent et de cadeaux d'un homme, sans lui accorder de faveurs sexuelles en retour - le risque de cette pratique étant le viol, mentionné comme le principal moyen de vengeance des hommes «victimes » des «detoothers » (Nyanzi et al., 2001; Amuyunzu-Nyamongo et al., 2005).

Les jeunes garçons ont aussi des motivations économiques et matérielles dans le choix de leurs partenaires, ce qui peut expliquer que certains décident d'en avoir plusieurs (Meekers et Calvès, 1997). La littérature sur ces expériences et sur les «sugar-mummies » est rare. En Ouganda, ce sont souvent des veuves dont le mari est décédé des suites de l'infection au VIH, qui n'arrivent pas à trouver un nouveau partenaire. En général, les jeunes gardent le secret de ce type de relations, ont une " petite amie » de leur âge et maintiennent la relation avec la «sugar-mummy » pour ses avantages économiques. Dans une étude menée entre autres au Ghana, l'évocation de l'expression "sugar-mummies » a provoqué une grande hilarité parmi les jeunes filles qui prenaient part à une 
discussion de groupe. Il ne s'agissait pas, indiquent les auteurs, d'une expression de gêne ou de malaise, mais de ridiculiser un jeune homme " faisant des choses qu'il ne devrait pas » (Amuyunzu-Nyamongo et al., 2005).

\section{Les constructions sociales de la masculinité et de la féminité}

Le comportement sexuel des adolescents est aussi déterminé par les constructions sociales de la masculinité et de la féminité, ce que Wood et al. (1998), Varga (2003), Eaton et al. (2003) ont bien montré pour l'Afrique du Sud. Chez les Zoulous d'Afrique du Sud, une jeune fille convenable doit être «disponible" pour son partenaire, lui être fidèle, éviter les grossesses et n'exprimer aucun désir sexuel ni prendre l'initiative. L'homme, quant à lui, est perçu comme un être ayant un besoin «naturel» de sexe, un désir sexuel irrépressible, ce qui rend acceptable le fait qu'il puisse avoir plusieurs partenaires. Cette idée d'un désir sexuel masculin irrépressible est très courante (Moore et Rosenthal, 1992 ; Nyanzi et al., 2001 ; Gavey et al., 2001), de même que celle qui associe la masculinité à des rapports sexuels non protégés avec plusieurs partenaires (Nyanzi et al. 2001; Eaton et al., 2003; Jewkes et al., 2003). En outre, un «vrai » homme doit être capable de vaincre les résistances, en employant si besoin la violence, pour satisfaire ses désirs ; une "vraie» femme doit, pour sa part, être fidèle, n'exprimer aucun désir, «s'offrir », «se laisser faire », tout en évitant les grossesses. Les filles doivent alors affronter ces contradictions, sachant que refuser une relation sexuelle peut donner lieu à des scènes de violence (Eaton et al., 2003).

Ces discours, qui privilégient les désirs sexuels des hommes, construisent la masculinité d'une façon qui affecte profondément l'expérience hétérosexuelle des femmes (Gavey et al., 2001) et reflètent une inégalité de pouvoir et de genre dont l'une des manifestations est la violence sexuelle ( «sexual coercion $\left.{ }^{15} »\right)$.

\section{La violence sexuelle}

La contrainte sexuelle consiste à forcer ou essayer de forcer, par la ruse, la violence, les paroles, les contraintes économiques, les normes sociales, etc., une personne à s'engager dans une activité sexuelle. Elle inclut les attouchements, le mariage forcé, les tentatives de viol, le viol. Les filles et les femmes en sont le plus souvent les victimes, affectées de manière inégale et les hommes, les auteurs. La violence ou le risque de violence sexuelle affectent la santé reproductive de diverses façons. Des études montrent une association entre violence physique et sexuelle et divers problèmes gynécologiques : saignements vaginaux,

\footnotetext{
15 Nous emploierons indifféremment les expressions «contrainte sexuelle» ou «violence sexuelle» pour «sexual coercion».
}

douleurs lors de rapports sexuels, infections urinaires, inflammations et douleurs pelviennes (Koenig et al., 2004). La violence ou la menace de violence limitent les capacités de négociation des comportements de prévention, notamment chez les femmes infectées par le VIH, qui ne peuvent dévoiler leur statut sans accroître encore ce risque (Maman et al., 2000 ; Jewkes et al., 2003). Des travaux montrent que les femmes séropositives mentionnent plus de violence physique et sexuelle que les femmes séronégatives (Koenig et al., 2004). On note une association entre la violence et les grossesses non désirées, entre la violence et une utilisation inconstante des contraceptifs. On note également une diminution de la probabilité d'utilisation des méthodes contraceptives et du préservatif chez les femmes qui ont préalablement été exposées à la violence sexuelle et physique, ou qui en ont peur. Les abus sexuels dans l'enfance et l'adolescence sont aussi associés ultérieurement à des comportements à risque.

Des enquêtes à l'échelle mondiale montrent que 10 à $20 \%$ de femmes ont été violentées par un partenaire et que 33 à $50 \%$ sont sujettes à des violences sexuelles (Gupta, 2002). Une étude au Nigeria révèle que, pour $20 \%$ des jeunes filles sexuellement actives, le premier rapport a été forcé (Ajuwon et al., 2001) ; au Ghana, sur 86 femmes sexuellement actives, $33 \%$ ont eu des rapports forcés (Afenyadu et Goparaju, 2003) ; au Cameroun, une étude menée auprès de 671 jeunes dans la province du NordOuest révèle que, pour $37 \%$ des filles et $30 \%$ des garçons, les premiers rapports sexuels n'ont pas été volontaires. En Afrique du Sud, la contrainte sexuelle fait partie de l'expérience de certaines adolescentes (Wood et al., 1998; Varga, 2003). Wood et al. (1998) relèvent un contrôle masculin de tous les aspects de l'expérience sexuelle de ces adolescentes qui n'ont, de façon générale, aucune idée du «sexe » avant leur première relation et sont donc «initiées » par les hommes. Cette ignorance fait de la première expérience, souvent forcée, une expérience traumatisante, marquée par une série d'injonctions : «undress », «lie on the bed», "open your legs». Le langage qu'utilisent les filles pour décrire leur expérience met en évidence la brutalité, la contrainte : «he just push me and overcame me», "he forced himself into me ", "he did as he wanted with me». Néanmoins, pour elles, il ne s'agit pas de viol, mais de signes de passion ou de stratégies pour se faire aimer. Ce paradoxe apparaît dans des expressions telles que : « he forced me to love him » ou «I fell in love with him because he beat me up ». Par la suite, plusieurs filles se complaisent aux désirs/demandes des hommes, n'imaginant pas que les rapports sexuels puissent apporter un quelconque plaisir.

Le mariage forcé, qui consiste à « donner » sa fille en mariage contre son gré, est une autre forme de violence sexuelle. Il intervient le plus souvent à l'âge de 13-14 ans, parfois moins, pour éviter un risque d'opposition (Guiella, 2004) ou parce qu'on estime qu'une fille doit être chez son 
mari avant la survenue des premières règles. En 2001, plus de 567 cas de refus de mariage forcé ont été enregistrés par la police de Fada N'gourma, une province située à environ $250 \mathrm{~km}$ de Ouagadougou (Guiella, 2004). Il est clair que ces chiffres ne représentent qu'une part de la réalité. Au Cameroun, dans le Nord et l'extrême Nord, les mariages forcés sont fréquents et la coordinatrice de l'Association de lutte contre les violences faites aux femmes (ALVF), qui soutient et accompagne les victimes, rappelait ${ }^{16} \mathrm{qu}^{\prime} \mathrm{il}$ n'est pas possible de mesurer l'ampleur du phénomène ni d'obtenir des statistiques fiables. Ne se présentent à l'association que celles qui se sont échappées du domicile conjugal et ne sont déclarées à la police que les femmes recherchées par la belle-famille ou les parents, ou bien celles qui ont porté plainte auprès de l'association. De celles qui ont été contraintes au mariage et qui demeurent encore chez leurs époux, on ne sait pas grand-chose et il est difficile d'obtenir des témoignages. On pourrait faire le même constat au sujet du viol. En Afrique du Sud, 45825 viols de femmes de tous les âges ont été déclarés à la police entre le $1^{\mathrm{er}}$ avril 2003 et le 31 mars 2004, soit un taux de viol de 194 pour 100000 femmes, trois fois plus élevé qu'aux États-Unis. Lorsque les recherches sont menées, les estimations sont plus élevées que les chiffres fournis dans les rapports de police. Ici, les hommes se servent du viol comme stratégie d'affirmation, de domination, d'expression de puissance. On pense aussi que violer une vierge peut guérir de l'infection par le VIH. Ces mêmes auteurs ont noté par la suite (Jewkes et al., 2006) que, pour échapper aux stigmates du viol, les victimes réinterprétaient l'acte sous divers formes (affection débordante du partenaire habituel, rapport sexuel forcé ?). Le viol peut être une action de groupe, considérée comme une sanction « légitime» envers une femme infidèle, une femme dont le comportement ne correspond pas aux normes de genre. Il peut aussi s'agir d'une «faveur » faite à des amis lorsqu'on veut rompre ou sceller un accord. Acte humiliant, ce type de viol ne donne lieu à aucune forme de réinterprétation. La honte, l'humiliation, l'absence de dispositif juste et efficace pour sanctionner les auteurs de viol sont autant de facteurs expliquant le silence, la réinterprétation de l'acte et l'absence de statistiques fiables. Les études sur cette question en Afrique concernent principalement les femmes. Pourtant, les garçons peuvent également en être victimes , mais ce type de violence est encore plus difficile à mesurer compte tenu du contexte socioculturel.

\section{Conclusion}

La littérature met en évidence la domination et la vulnérabilité des adolescentes, attirées par les «sugar-daddies", dominées par un discours sur la sexualité qui se concentre

\footnotetext{
16 Entretien personnel, août 2006.
}

sur le désir masculin, violentées «par amour ». Toutefois, nous pensons, comme Wojcicki et Malala (2001), qu'on ne doit pas exclusivement considérer les jeunes femmes comme des victimes. Si elles ne sont pas «empowered» dans le sens d'être en mesure d'orienter leur vie en fonction des opportunités, elles prennent des décisions, font des choix, ont même à un niveau microsocial des initiatives ayant une incidence réelle, positive ou négative, sur leur vie.

Les grossesses non désirées et les IST posent des problèmes sociaux réels. Cependant, une focalisation exclusive sur les facteurs de risque tend à faire oublier le contexte. Le comportement sexuel des adolescents est tributaire des autres aspects de leur vie, de l'environnement social, économique et culturel dans lequel ils évoluent. Ainsi, l'usage ou non du préservatif ne peut se comprendre sans prendre en compte les éléments qui investissent le champ des rapports hétérosexuels : les contraintes économiques, les constructions sociales de la masculinité et de la féminité avec les inégalités de pouvoir et la violence qu'elles supposent, la disponibilité du préservatif, les contraintes liées à son utilisation.

\section{Références}

Afenyadu, D., Goparaju, L., 2003. Adolescent Sexual and Reproductive Health Behaviour in Dodowa, Ghana, Washington, USAID/CEDPA.

Ajuwon, A.J., Akin-Jimoh, I., Olley Iladapo, B., Akintola, O., 2001. Perceptions of sexual coercion: Learning from young people in Ibadan, Nigeria, Reproductive Health Matters, 9, 17, 128-136.

Amuyunzu-Nyamongo, M., Biddlecom, A.E., Ouedraogo, C., Woog, V., 2005. Qualitative Evidence on Adolescents Views of Sexual and Reproductive Health in sub-Saharan Africa. Occasional Report, The Alan Guttmacher Institute, New York and Washington.

Bankole, A., Singh, S., Woog, V., Deirdre, W., 2004. Risk and Protection: Youth and HIV/AIDS in sub-Saharan Africa, The Alan Guttmacher Institute, New York and Washington.

Barker, K.G., Rich, S., 1992. Influences on adolescent sexuality in Nigeria and Kenya: Findings from recent focus group discussion, Studies in Family Planning, 23, 3, 199-210.

Bearinger, L., Sieving, R., Ferguson, J., Sharma, V., 2007. Global perspectives on the sexual and reproductive health of adolescents: Patterns, prevention and potential, The Lancet, 369, 7, 1220-1231.

Brooks-Gunn, J., Furstenberg, F., 1990. Coming of age in the era of AIDS: Puberty, sexuality and contraception, The Milbank Quaterly, 68, Suppl. 1, Part 1, 59-84.

Caldwell, J., Caldwell, P., Quigging, P., 1989. The social context of AIDS in sub-Saharan Africa, Population and Development Review, 15, 2, 185-234.

Calvès, A.-E., 1999. The marginalization of African Single mothers in the marriage market: evidence from Cameroon, Population Studies, 13, 3, 291-301.

Calvès, A.-E., 2002. Abortion risk and decisionmaking among young people in urban Cameroon, Studies in Family Planning, $33,3,249-260$. 
Cameron, K., Salazar, L., Jay, B., Burgess-Whitman, N., Wingood, G., DiClemente, R., 2005. Adolescents experience with sex on the web: Results from online focus groups, Journal of Adolescence, 28, 535-540.

Dehne, K., Riedner, G., 2001. Adolescence: A dynamic concept, Reproductive Health Matters, 9, 17, 11-15.

Dowsett, G., Aggleton, P., 1999. Sex and Youth: Contextual Factors Affecting Risk for HIV/AIDS. A Comparative Analysis of Multi-Site Studies in Developing Countries, Geneva, UNAIDS. Document téléchargeable sur : http://data.unaids.org/ Publications/IRC-pub01/jc096-sex|\%26youth-2_en.pdf

Eaton, L., Flisher, A., Leif, A., 2003. Unsafe sexual behavior in South African youth, Social Science and Medicine, 56, 149-165.

Erulkar, A., Mekbib, T-A., Simie, N., Gulema, T., 2006. Differential use of adolescent reproductive health programs in Addis Ababa, Ethiopia, Journal of Adolescent Health, 38, 253-260.

Gage, A.J., 1998. Sexual activity and contraceptive use: The components of the decisionmaking process, Studies in Family Planning, 29, 2, 154-166.

Gavey, N., MacPhillips, K., Doherty, M., 2001. "If it's not on, it's not on": or is it? Discursive constraints on women's condom use, Gender and Society, 15, 6, 917-934.

Gazmararian, J., Petersen, R., Spitz, A.M., Goodwin, M.M., Saltzman, L.E., Marks, J.S., 2000. Violence and reproductive health: Current knowledge and future research directions, Maternal and Child Health Journal, 4, 2, 79-84.

Geronimus, A., 1991. Teenage childbearing and social and reproductive disadvantage: The evolution of complex questions and the demise of simple answers, Family Relations, 40, 4, 463-471.

Geronimus, A., 1992. Teenage childbearing and social disadvantage: Unprotected discourse, Family Relations, 41, 2, 244-248.

Geronimus, A., 2004. Teenage childbearing as cultural prism, British Medical Bulletin, 69, 155-166.

Glasier, A., Gülmezoglu, A., 2006. Putting sexual and reproductive health on the agenda, The Lancet, 368, 9547, 1550-1551.

Gorgen, R., Maier, B., Diesfeld, H.J., 1993. Problems related to schoolgirl pregnancies in Burkina Faso, Studies in Family Planning, 24, 5, 283-294.

Gueye, M., Castle, S., Konate, M., 2001. Timing of first intercourse among Malian adolescents: implications for contraceptive use, International Family Planning Perspectives, 27, 2, 56-62.

Guiella, G., 2004. Santé sexuelle et de la reproduction des jeunes au Burkina Faso: un état des lieux. Occasional Report, № 12, The Alan Guttmacher Institute, New York and Washington.

Gupta, R., 2002. How men's power over women fuels the HIV epidemic, British Medical Journal, 324, 183-184.

Hanna, B., 2001. Adolescent parenthood: A costly mistake or a search for love?, Reproductive Health Matters, 9, 17, 101-107.

Harrison, A., Xaba, N., Kunene, P., 2001. Understanding safe sex: gender narratives of HIV and pregnancy prevention by rural South African School-going youth, Reproductive Health Matters, 9, 17, 63-71.

Horton, R., 2006. Reviving reproductive health, The Lancet, 368, 1548-1549.

Jewkes, R., Levin, J., Penn-Kekana, L., 2003. Gender inequalities, intimate partner violence and HIV preventive practices: Findings of a South African cross-sectional study, Social Science and Medicine, 56, 125-134.

Jewkes, R., Dunkle, K., Koss, M., Levin, J., Nduna, M., Jama, N., Sikweyiya, Y., 2006. Rape perpetration by young, rural
South African men: Prevalence, patterns and risk factors, Social Science and Medicine, 63, 2949-2961.

Kau, M., 1991. Sexual behaviour and knowledge of adolescent males in the Molopo Region of Bophuthatswana, Curationis, 14, 1, 37-40.

Kim, J., Watts, C., 2005. Gaining foothold: Tackling poverty, gender inequality, and HIV in Africa, British Medical Journal, 331, 769-772.

Koenig, M., Zablotska, I., Lutalo, T., Nalugoda, F., Wagman, J., Gray, R., 2004. Coerced first intercourse and reproductive health among adolescent women in Rakai, Uganda, International Family Planning Perspectives, 30, 4, 156-163.

Lallemant, M., Jourdain, G., 1985. La grossesse impossible chez les jeunes filles scolarisées à Brazzaville, Cahiers de l'ORSTOM, série Sciences humaines, XXI, 2-3, 363-372.

Langer, A., 2006. Cairo after 12 years: Successes, setbacks, and challenges, The Lancet, 368, 1552-1553.

LeGrand, T., Mbacké, C., 1993. Teenage pregnancy and child health in the urban Sahel, Studies in Family Planning, 24, 3 , 137-149.

Luke, N., 2005. Confronting the "Sugar Daddy" stereotype: Age and economic asymmetries and risky sexual behavior in urban Kenya, International Family Planning Perspectives, 31, 1, 6-14.

Macleod, C., 2002. Economic security and the social science literature on teenage pregnancy in South Africa, Gender and Society, 16, 5, 647-664.

MacPhail, C., Campbell, C., 2001. "I think condoms are good but, aai, I hate those things": Condom use among adolescents and young people in a Southern African township, Social Science and Medicine, 52, 1613-1627.

Maina Ahlberg, B., 1994. Is there a distinct African sexuality? A critical response to Caldwell, Africa, Journal of the International African Institute, 64, 2, 220-242.

Maina Ahlberg, B., Jylkäs, E., Krantz, I., 2001. Gendered construction of sexual risks: Implications for safer sex among young people in Kenya and Sweden, Reproductive Health Matters, 9, 17, 26-36.

Maman, S., Campbell, J., Sweat, M., Gielen, A., 2000. The intersections of HIV and violence: directions for future research and interventions, Social Science and Medicine, 50, 459-478.

Marshall, D.S., Suggs, R.C., 1970. Human Sexual Behaviour: Variations in the Ethnographic Spectrum, New York, Basic Books.

Meekers, D., Calvès, A.-E., 1997. "Main" girlfriends, girlfriends, marriage and money: The social context of HIV risk behaviour in sub-Saharan Africa, Health Transition Review, Supp. to vol. 7, 361-375.

Meyer-Weitz, A., Reddy, P., Van Den Borne, W., Kok, G., Pietersen, J., 2000. The determinants of health care seeking behaviour of adolescents attending STD clinics in South Africa, Journal of Adolescence, 23, 741-752.

Moore, S., Rosenthal, D., 1992. The social context of adolescent sexuality: Safe sex implications, Journal of Adolescence, 15, 4, 415-435.

Nyanzi, S., Pool, R., Kinsman, J., 2001. The negotiation of sexual relationships among school pupils in South-Western Uganda, AIDS Care, 13, 1, 83-98.

ONUSIDA, OMS, 2007. Le Point sur l'épidémie de sida. Rapport spécial sur la prévention du VIH, ONUSIDA/07.27F/JC1322F, Genève. Document téléchargeable à l'adresse Internet : http://www.unaids.org/fr/KnowledgeCentre/HIVData/ EpiUpdate/EpiUpdArchive/2007/default.asp 
Reynolds, H.D., Wong, E.L., Tucker, H., 2006. Adolescents use of maternal and child health services in developing countries, International Family Planning Perspectives, 32, 1, 6-16.

Rwenge, M., 2000. Sexual risk behaviors among young people in Bamenda, Cameroon, International Family Planning Perspectives, 26, 3, 118-130.

Sariola, H., Uutela, A., 1994, The prevalence of child sexual abuse in Finland, Child Abuse and Neglect, 18, 10, 827-835

Scheper-Hughes, N., 1994. Aids and the social body, Social Science and Medicine, 39, 7, 991-1003.

Schwab Zabin, L., Kiragu, K., 1998. The health consequences of adolescent sexual and fertility behavior in sub-Saharan Africa, Studies in Family Planning, 29, 2, 210-232.

Scott-Sheldon, L., Glasford, D., Marsh, K., Lust, S., 2006. Barriers to condom purchasing: Effects of product positioning on reactions to condoms, Social Science and Medicine, 63, 27552769.

Shoveller, J., Johnson, J., Langille, D., Mitchell, T., 2004. Sociocultural influences on young people's sexual development, Social Science and Medicine, 59, 473-487.

Singer, M., Erickson, P., Badiane, L., Diaz, R., Ortiz, D., Abraham, T., Nicolaysen, A., 2006. Syndemics, sex and the city: Understanding sexually transmitted diseases in social and cultural context, Social Science and Medicine, 63, 2010-2021.

Singh, S., 1998. Adolescent childbearing in developing countries: A global review, Studies in Family Planning, 29, 2, 117-136.

Speizer, I., Tambashe, O., Tegang, S-P., 2006. An evaluation of the "entre nous jeunes" peer-educator program for adolescents in Cameroon, Studies in Family Planning, 32, 4, 339-351.

Standing, H., 1992. AIDS: Conceptual and methodological issues in researching sexual behaviour in sub-Saharan Africa, Social Science and Medicine, 34, 5, 475-483.

Reçu le 7 février 2007. Accepté le 6 novembre 2008.
Sumartojo, E., 2000. Structural factors in HIV prevention: Concepts, example, and implications for research, AIDS, 14, Supp. 1, S3-S10.

Sumartojo, E., Doll, L., Holtgrave, D., Gayle, H., Merson, M., 2000. Enriching the mix: Incorporating structural factors into HIV prevention, AIDS, 14, Supp. 1, S1-S2.

Swart-Kruger, J., Richter, L., 1997. Aids-related knowledge, attitudes and behaviour among south African street youth: Reflections on power sexuality and the autonomous self, Social Science and Medicine, 45, 6, 957-966.

Tolman, D. L., 1994. Doing desire. Adolescent girl's struggles for/with sexuality, Gender and Society, 8, 3, 324-342.

Varga, C., 2001. The forgotten fifty per cent: A review of sexual and reproductive health research and programs focused on boys and young men in sub-Saharan Africa, African Journal of Reproductive Health, 5, 3, 175-195.

Varga, C., 2003. How gender roles influence sexual and reproductive health among South African adolescents, Studies in Family Planning, 34, 3, 160-172.

WHO, 1998. The Second Decade: Improving Adolescent Health and Development, Geneva, WHO.

Wojcicki, J.M., Malala, J., 2001. Condom use, power and HIV/AIDS risk: Sex workers bargain for survival in Hillbrow/Joubert Park/Bera, Johannesburg, Social Science and Medicine, 53, 1, 99-121.

Wood, K., Maforah, F., Jewkes, R., 1998. "He forced me to love him": Putting violence on adolescent sexual health agendas, Social Science and Medicine, 47, 2, 233-242.

Zellner, S., 2003. Condom use and the accuracy of AIDS knowledge in Cote d'Ivoire, International Family Planning Perspectives, 29, 1, 41-47. 